\title{
Time-domain terahertz optoacoustics: manipulable water sensing and dampening
}

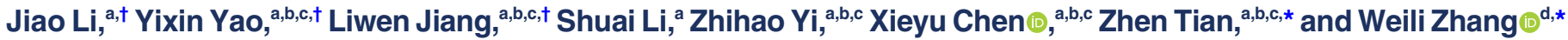 \\ ${ }^{a}$ Tianjin University, School of Precision Instruments and Optoelectronics Engineering, Tianjin, China \\ ${ }^{b}$ Tianjin University, Center for Terahertz Waves, Tianjin, China \\ 'Ministry of Education, Key Laboratory of Optoelectronics Information and Technology, Tianjin, China \\ dOklahoma State University, School of Electrical and Computer Engineering, Stillwater, Oklahoma, United States
}

\begin{abstract}
Radiation at terahertz frequencies can be used to analyze the structural dynamics of water and biomolecules, but applying the technique to aqueous solutions and tissues remains challenging since terahertz radiation is strongly absorbed by water. While this absorption enables certain analyses, such as the structure of water and its interactions with biological solutes, it limits the thickness of samples that can be analyzed, and it drowns out weaker signals from biomolecules of interest. We present a method for analyzing water-rich samples via time-domain terahertz optoacoustics over a $10^{4}$-fold thickness ranging from microns to centimeters. We demonstrate that adjusting the temperature to alter the terahertz optoacoustic ( $\mathrm{THz}-\mathrm{OA})$ signal of water improves the sensitivity with which it can be analyzed and, conversely, can reduce or even "silence" its signal. Temperature-manipulated THz-OA signals of aqueous solutions allow detection of solutes such as ions with an order of magnitude greater sensitivity than terahertz time-domain spectroscopy, and potentially provide more characteristic parameters related to both terahertz absorption and ultrasonic propagation. Terahertz optoacoustics may be a powerful tool for spectroscopy and potential imaging of aqueous solutions and tissues to explore molecular interactions and biochemical processes.
\end{abstract}

Keywords: terahertz optoacoustics; terahertz pulse; time-domain detection; label-free; water.

Received Dec. 12, 2020; revised manuscript received Feb. 3, 2021; accepted for publication Mar. 1, 2021; published online Apr. 1, 2021.

(C) The Authors. Published by SPIE and CLP under a Creative Commons Attribution 4.0 Unported License. Distribution or reproduction of this work in whole or in part requires full attribution of the original publication, including its DOI.

[DOI: 10.1117/1.AP.3.2.026003]

\section{Introduction}

Terahertz radiation, with wavelengths between 0.03 and $0.3 \mathrm{~mm}$, can provide unique insights into the composition, structure, and dynamics of inorganic and biological materials unavailable with radiation of other frequencies..$^{1-5}$ It can detect numerous rotational and vibrational transitions in proteins, DNA, RNA, and sugars, ${ }^{6-10}$ as well as transitions in water that depend on hydrogen bonding, which means that it is sensitive to interactions of water with solutes such as ions. ${ }^{11-18}$ The extremely low energy of terahertz radiation makes it safe for analysis of living samples. Because of these advantages, terahertz spectroscopy and imaging have developed into an

*Address all correspondence to Zhen Tian, tianzhen@tju.edu.cn; Weili Zhang, weili.zhang@okstate.edu

tThese authors contributed equally to this work. effective technique for detecting proteins, DNA, RNA, sugars, and cells in vitro and in vivo, ${ }^{9,10,19-23}$ for analyzing structures and dynamics of water and ion hydration, ${ }^{18,24-26}$ for differentiating tumors from healthy cells based on water content, ${ }^{27-31}$ and for evaluating tissues for the presence of viruses or other pathology. ${ }^{32-34}$

In this way, the strong absorption of terahertz radiation by water enables a range of analyses. On the other hand, it prevents other types of analysis, where the strong absorption drowns out absorption by the molecules of interest. To reduce this background, terahertz reflectance spectra or attenuated total reflectance spectra are obtained, or extremely thin samples are used. ${ }^{35-37}$ Alternatively, researchers have attempted to boost the desired signal by increasing the intensity of terahertz radiation $^{38}$ or the sensitivity of terahertz detectors. ${ }^{39}$ These solutions may be unsatisfactory in the long run because water background exponentially attenuates the desired signal, ultrahigh 
intensity radiation brings health risks, ${ }^{40,41}$ and ultrasensitive detectors can be prohibitively expensive.

We wondered whether we could induce water itself to be a detector for interactions between terahertz radiation and waterrich samples, such as aqueous solutions and tissues. Relying on the water within the sample, rather than on external terahertz detectors to capture the terahertz radiation emitted from the surface of the sample, would avoid the limitation on measurement depth due to the strong absorption of water. Upon absorption of terahertz radiation, aqueous media heat up, inducing thermoelastic expansion that produces acoustic waves that can be detected, which is referred to as the opto- or photoacoustic effect. ${ }^{40-42}$ So far, efforts on time-domain terahertz optoacoustics have relied on carbon nanotube nanocomposites. ${ }^{42}$ Terahertz optoacoustic (THz-OA) studies in water have shown that destructive shockwaves, which are generated by strong terahertz radiation, can traverse a millimeter-thick aqueous solution. ${ }^{40,41}$ However, this THz-OA effect of water using terahertz pulses with excessively high repetition rate or high pulse energy is not suitable for time-domain measurement and nondestructive precise testing in biomedical applications. In addition, the strong absorption of water in the terahertz regime drowns out the signal from much less abundant but biologically important molecules such as proteins, glucose, or hydrated ions. Therefore, a revolutionary time-domain $\mathrm{THz}-\mathrm{OA}$ method that could detect not only water but also much less abundant biomolecules in native samples using ultrashort terahertz pulses with safe radiation intensities is much desired in various fields, particularly in biochemistry and biomedicine.

In this article, we present time-domain terahertz optoacoustics in analyzing water-rich samples, which enables water itself to be induced as a detector that directly detects the interactions between terahertz radiation and water in aqueous solutions and tissues. We demonstrate the significant potential of this method using agar-in-water phantoms, ex vivo tissues, as well as water and aqueous solutions in microfluidic chips. The sample temperature can be controlled to manipulate time-domain THz-OA signals from water, thereby either enhancing the sensitivity of water detection or dampening the water signal to detect signals from solutes of interest. Our findings may provide a basis for new methods and technologies in the terahertz regime, such as THzOA spectroscopy and imaging of aqueous and biological systems to study molecular interactions and biochemical processes.

\section{Method}

\subsection{Experimental Setup and Data Acquisition}

The THz-OA system presented here incorporates several unique characteristics that maximize the THz-OA signal from native tissues or aqueous solutions, while also allowing the dampening of signals from water to highlight signals from biomolecules of interest [Fig. 1(a)]. The terahertz pulses were generated using a femtosecond Ti:sapphire regenerative amplifier (Coherent) at a wavelength of $800 \mathrm{~nm}$ and energy of $5 \mathrm{~mJ}$ to pump the nonlinear crystal $\mathrm{LiNbO}_{3}$ using the tilted-pulse-front technique [Fig. 1(a)]. ${ }^{43}$ The pump laser beam had a pulse duration of $35 \mathrm{fs}$ and a repetition rate of $1 \mathrm{kHz}$. A pulse front tilt of $63 \mathrm{deg}$ was introduced using a reflective grating and a cylindrical lens. To increase terahertz field strength per unit area, off-axis parabolic mirrors were used to collimate and focus the terahertz beam to a spot of $\sim 1.5 \mathrm{~mm}$ at the center of the sample holder with

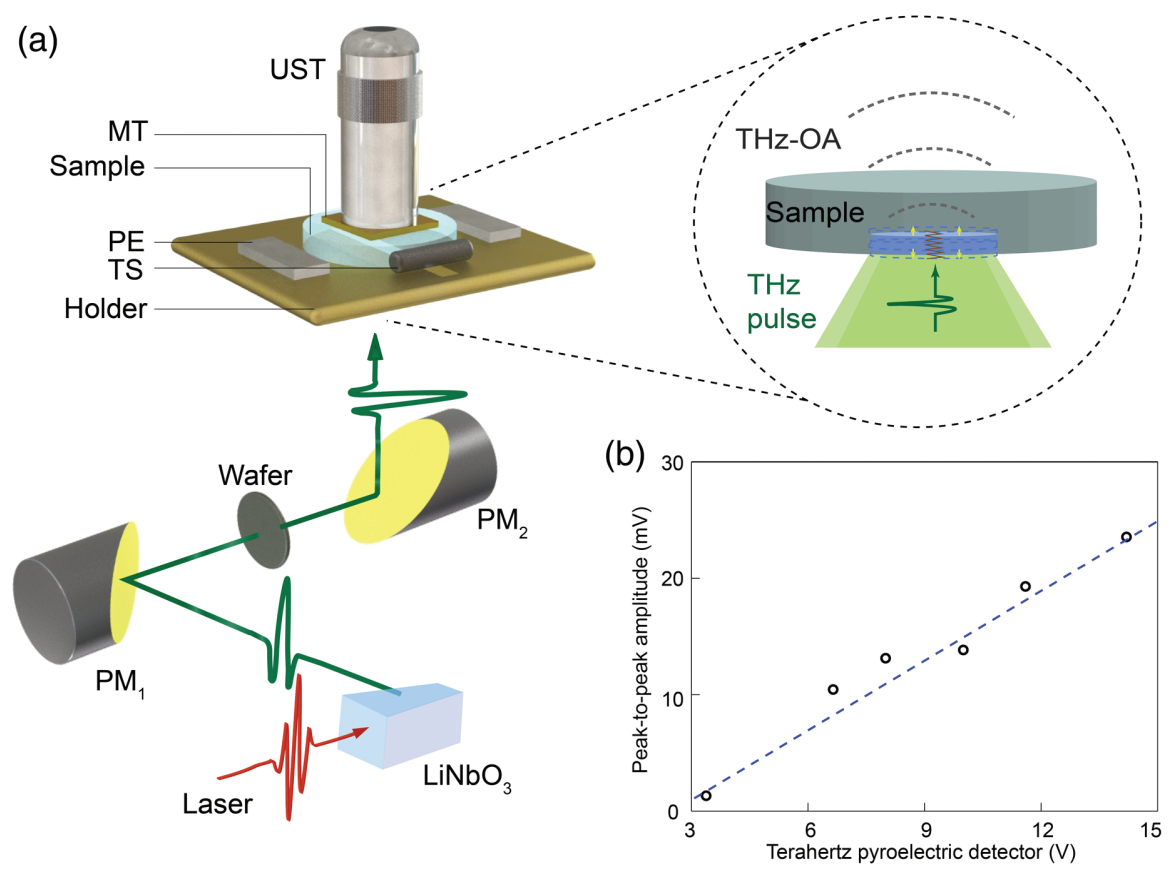

Fig. 1 Schematic for time-domain THz-OA measurement. (a) Schematic of the setup. MT, metal tape; $\mathrm{PM}_{1,2}$, parabolic mirrors; $\mathrm{PE}$, Peltier element; THz-OA, terahertz optoacoustic; TS, temperature sensor; UST, ultrasonic transducer. The light blue region represents the interaction area between the terahertz radiation and sample. (b) Amplitude of THz-OA signal from water measured by the UST as a function of the energy of the terahertz radiation measured by a terahertz pyroelectric detector. The best-fit line (dotted blue) is also shown. 
the energy per pulse of $4 \mu \mathrm{J}$ and spectral range of 0.2 to $1.5 \mathrm{THz}$. The radiant exposure is $0.23 \mathrm{~mJ} / \mathrm{cm}^{2}$, which satisfies the confinement conditions for optoacoustics ${ }^{44}$ and falls within the laser exposure limit of $20 \mathrm{~mJ} / \mathrm{cm}^{2} .{ }^{45,46}$ A silicon wafer with a 4 in. diameter and $1 \mathrm{~mm}$ thickness, whose surfaces on both sides are pasted with 0.1 -mm-thick black polyethylene sheet, is used to block the stray light of the pump laser, thereby preventing interference with the THz-OA signal. An IR Night Vision device (84499A, FJW) is employed to ensure that there is no stray light at $800 \mathrm{~nm}$ illuminating the samples.

The signals were detected using flat piezoelectric ultrasonic transducers (Olympus) with central frequencies of 1 to $2.5 \mathrm{MHz}$, according to the frequency range of simulated $\mathrm{THz}-\mathrm{OA}$ signals of water (Fig. S4 in Supplementary Material). The signals were then amplified by a low-noise 50-dB amplifier (Usultratek), digitized at a sampling rate of $200 \mathrm{MS} / \mathrm{s}$ using a data acquisition card (Gage), and stored in a PC. The detection signal-to-noise ratio was enhanced by averaging every 1000 pulses. The THzOA signal detected from a sample of pure water varied linearly with the amplitude detected using a traditional terahertz pyroelectric method [Fig. 1(b)].

Microfluidic chips were custom-built to have a rear surface of polydimethylsiloxane (PDMS) $(5 \mathrm{~mm}$ thick) and a front surface of PDMS (200 $\mu \mathrm{m})$ or quartz (300 $\mu \mathrm{m})$ [Fig. 2(d)]. The chip's detection area was a circle with a diameter that was $3 \mathrm{~mm}$ larger than the terahertz-focused area. The temperature of the

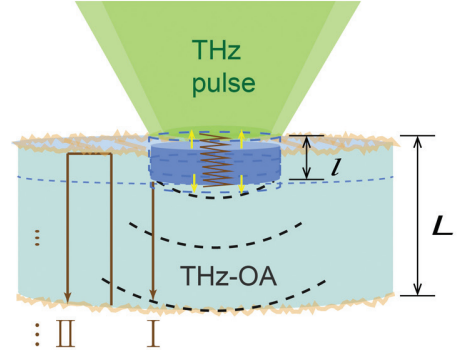

(a)

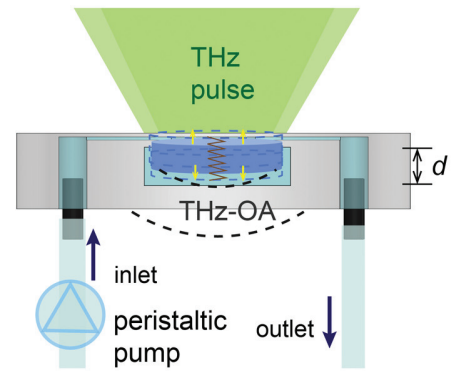

(d)

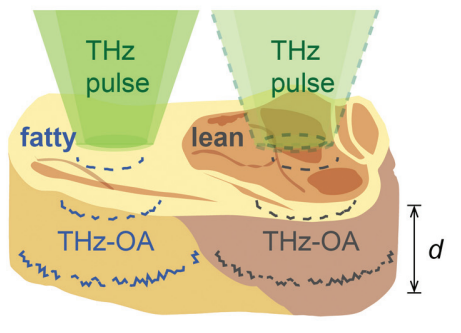

(g)

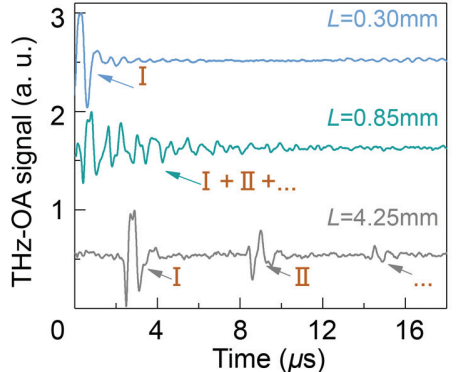

(b)

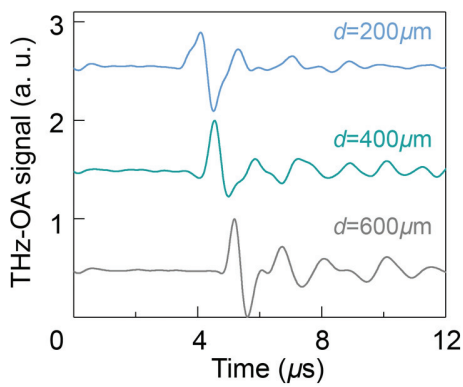

(e)

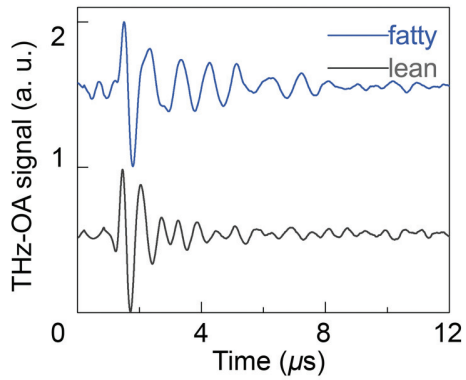

(h)

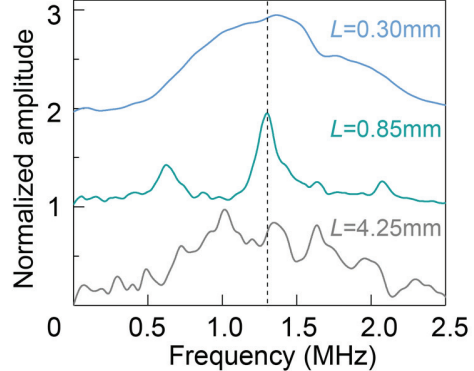

(c)

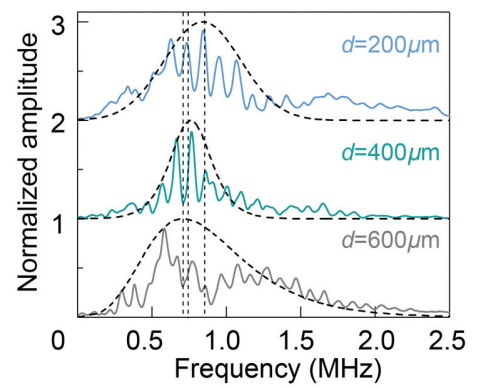

(f)

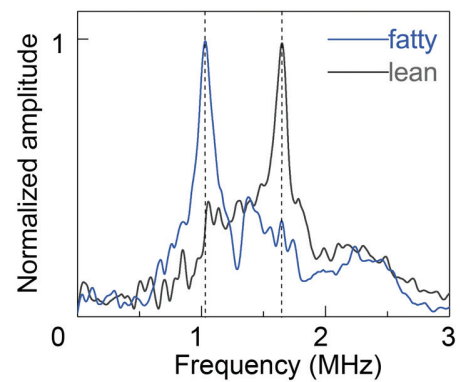

(i)

Fig. 2 THz-OA responses from an agar-in-water phantom, water flowing through a microfluidic chip, and a fresh beef brisket slice. (a) Schematic of the production of THz-OA signal by an agar phantom of thickness $L$ after terahertz irradiation to a penetration depth I. I, II, and ... denote the primary THz-OA signal and its echoes. (b) THz-OA signals in the time domain for three $L$ values. (c) First time-domain $\mathrm{THz}-\mathrm{OA}$ signals from (b) were transformed to the frequency domain. (d) Schematic of the production of THz-OA signal by water circulating through a channel of depth $d$ on a microfluidic chip. A peristaltic pump drives water into the sample holder at the inlet, and the water exits at the outlet. (e) THz-OA signals in the time domain for three $d$ values. (f) THz-OA signals from (e) were transformed to the frequency domain. $(\mathrm{g})$ Schematic of the production of THz-OA signal by ex vivo tissue, with the fatty and lean areas of interest marked. (h) Time-domain $\mathrm{THz}-\mathrm{OA}$ signals from each area of interest. (i) $\mathrm{THz}-\mathrm{OA}$ signals from (h) were transformed to the frequency domain. 
sample within the chip was controlled using a custom-built control module [Fig. 1(a)]. Two Peltier elements, the temperature sensor, and the chip were fixed atop a copper holder $[401 \mathrm{~W} /(\mathrm{m} \cdot \mathrm{K})]$ using thermoconductive adhesive. Care was taken to align the center of the chip with the narrow slit ( $3 \mathrm{~mm})$ in the center of the holder in order to ensure interaction with the strongest part of the terahertz field. The temperature controller applied heating or cooling based on the difference between the preset temperature and the sensor's measurement. The temperature sensor measured the holder as a proxy for the sample. The liquid sample was pushed through the chip at constant speed using a peristaltic pump [Fig. 2(d)] in order to prevent bubble formation due to the temperature change.

The rear surface of the sample was covered with metal tape [Fig. 1(a)] to prevent terahertz radiation from hitting the transducer directly, while still allowing for the arrival of acoustic waves from the sample. Ultrasound gel was added as a coupling agent between the sample and transducer. As a benchmark for our THz-OA measurements, we also analyzed samples using a commercially available terahertz time-domain spectroscopy (THz-TDS) system (T-Ray 5000, API Picometrix).

\subsection{Samples}

Cylindrical agar phantoms were prepared by mixing agar powder and distilled water in a mass ratio of 2.8:100. Phantom thickness $L$ [Fig. 2(a)] was measured to an error $<100 \mu \mathrm{m}$ using a Vernier caliper. Fresh meats were obtained from a local butcher's shop and cut into slices $1000 \pm 200 \mu \mathrm{m}$ thick. Aqueous solutions of glucose and ions were prepared using sterile deionized water.

\subsection{Basic Principles of Terahertz Optoacoustics}

Illuminating an absorber with a short electromagnetic pulse leads to an initial optoacoustic pressure $P_{0}$ described by ${ }^{47}$

$P_{0}=\Gamma \eta_{\mathrm{th}} \mu_{a} F=\left(\frac{\beta V_{S}^{2}}{C_{P}}\right) \eta_{\mathrm{th}} \mu_{a}(\lambda)$,

where $\Gamma$ is the dimension-less Grüneisen parameter, which represents the anharmonicity of the absorption material and is related to the thermal coefficient of volume expansion $\beta$, the speed of sound $V_{S}$, and the specific heat capacity $C_{P}$. The factor $\mu_{a}$ represents the optical absorption coefficient, $F$ shows the optical fluence (the optical energy per unit area), and $\eta_{\text {th }}$ defines the percentage of absorbed energy converted into heat, which is usually characteristic for a given material but is generally considered to be $1 .^{48} \Gamma$ depends only on the absorbing material, while $F$ depends on the characteristics of the electromagnetic wave. The parameter $\mu_{a}$ is determined by the absorption characteristics of the material at the given frequency of the electromagnetic wave. ${ }^{49}$

In aqueous solutions, the parameters $\mu_{a}, \beta, V_{S}$, and $C_{P}$ are functions of both temperature and solute concentration. The optoacoustic signals of glucose solutions with different concentrations (see Fig. S3 in Supplementary Material) were simulated using k-Wave software ${ }^{50}$ and parameters from the literature. ${ }^{51-53}$

The initial acoustic pressure is generated according to Eq. (1) and traverses the samples to the transducer. ${ }^{54}$ The detected signal will be affected by the spatial impulse response (SIR) and the electronic impulse response (EIR) of the transducer. ${ }^{48,55}$ The SIR is related to the shape and location of the transducer surface. The EIR is related to the properties of the piezoelectric crystal and the probe circuit. The final detected pressure can be expressed as $P=P_{0}(r, t) \otimes \operatorname{SIR}(r, t) \otimes \operatorname{EIR}(t)$.

\section{Results}

First, we tested the detection of the time-domain THz-OA signals from water in different samples of agar-in-water phantoms, water in microfluidic chips, and ex vivo tissues to verify the feasibility of using water as a detector for terahertz radiation. In particular, we examine how the detected signals would change with the distance $l$ traveled by the terahertz pulses to the terahertz absorber, corresponding to the "penetration depth." We varied the total thickness $L$ of agar-in-water phantoms, allowing it to range from smaller to much larger than the maximum penetration depth $l_{\max }$ [Fig. 2(a)]. When $L>l_{\max }$, we expected that the mismatch of the acoustic impedance between air and agar would cause the primary THz-OA signal (I) to be reflected back and forth between two surfaces within the phantom, generating multiple echoes (II, ...) [traces marked with brown arrows, Fig. 2(a)]. As expected, the THz-OA signals varied directly with $L$, in both the time domain [Fig. 2(b)] and frequency domain [Fig. 2(c)]. Analysis in the time domain showed that when $L=0.3 \mathrm{~mm}\left(L \leq l_{\max }\right)$, only one typical $\mathrm{N}$-shaped signal was observed [Fig. 2(b)], indicating transmission of the acoustic wave to the detector without an initial delay or echo. When $L=$ $0.85 \mathrm{~mm}\left(L>l_{\max }\right)$, the signal showed a small initial delay and overlap between the primary signal (I) and its echoes. When $L=4.25 \mathrm{~mm}\left(L \gg l_{\max }\right)$, the original signal (I) and multiple echoes $(\mathrm{II}, \ldots$ ) of similar shape and decreasing amplitude were observed, with increasing initial delay. Analysis in the frequency domain showed that when $L \leq l_{\max }$, the signal had the widest acoustic frequency band [Fig. 2(c)]. When $L>l_{\max }$, the signal overlap strengthened certain frequencies, including the central frequency of $\sim 1.3 \mathrm{MHz}$, as a result of resonance. When $L \gg l_{\max }$, the signal in the frequency domain showed no obvious central peak but several attenuated highfrequency components due to acoustic attenuation with the increase of the ultrasonic transmission path. The phantom experiments demonstrate that the proposed time-domain $\mathrm{THz}$ OA method avoids the thickness limitation on aqueous samples when measuring terahertz transmission.

Next, we analyzed the variation in water's THz-OA signal with varying sample thickness using a microfluidic chip, where we could accurately manipulate the penetration depth by altering the channel depth $d$ [Fig. 2(d)]. A deeper channel means a longer ultrasonic transmission path and therefore longer initial delay in the signal [Fig. 2(e)]. With increasing channel depth, the signal shifted toward lower frequencies, as the THz-OA signal broadened due to deeper penetration, and the high-frequency components were attenuated due to the longer ultrasonic transmission path [Fig. 2(f)]. These experiments demonstrate that liquid water can be directly induced to sense terahertz radiation, and its thickness can be precisely manipulated to study the interaction between terahertz radiation and liquid water without attenuation of detection sensitivity.

To validate nondestructive THz-OA testing on biological samples, we applied this to slices $\sim 1 \mathrm{~mm}$ thick of fresh beef brisket ex vivo [Fig. 2(g)]. The THz-OA signals were obtained from one fatty region and one lean region. Analysis in the time domain showed that signals from neither region penetrated the 
entire tissue and that signals from the fatty region were wider and lasted longer than signals from the lean region [Fig. 2(h)]. Analysis in the frequency domain showed that signals centered around $\sim 1.7 \mathrm{MHz}$ from the fatty region and $\sim 1 \mathrm{MHz}$ from the lean region [Fig. 2(f)]. This difference is consistent with the higher lipid content and therefore lower water content of fat, which results in deeper penetration that generates lowerfrequency acoustic signals. Similar results were obtained with various other types of lean and fatty meats (see Fig. S1 in Supplementary Material). The ex vivo experiments demonstrate that tissues differing in biomolecular composition produce distinguishable THz-OA signals in the time and frequency domains, suggesting the potential for biomedical applications.

The experiments with agar-in-water phantoms, water in a microfluidic chip, and tissues ex vivo demonstrate that the proposed THz-OA effect can directly induce water to act as a detector of terahertz radiation, even in thick samples. Next, we will examine whether the time-domain THz-OA method could manipulate the optoacoustic signal from water to enhance the sensitivity of water-based sensing or dampen it to allow sensitive detection of other molecules of interest. We hypothesized that we could manipulate the signal through temperature, as shown previously for water and aqueous solutions in optoacoustic spectroscopy in the megahertz regime. ${ }^{56}$ In the experiment, a temperature control module was utilized, and a peristaltic pump delivers liquid into the channel of the microfluidic chip on the sample holder at a defined temperature [Figs. 1(a) and 2(d)]. When pure water was characterized, the THz-OA signal showed the amplitude that varied directly with temperature over the biologically relevant range from $0^{\circ} \mathrm{C}$ to $40^{\circ} \mathrm{C}$. This result was observed in the time domain [Fig. 3(a)] and frequency domain [Fig. 3(b)], with amplification exceeding 10-fold over the temperature range. Closer analysis revealed that amplitude first decreased from $0^{\circ} \mathrm{C}$ to around $2^{\circ} \mathrm{C}$, after which it increased [Fig. 3(c)]. Therefore, under our experimental conditions, the thermal expansion coefficient or Grüneisen parameter of water fell to zero, and the minimum THz-OA signal was observed around $2^{\circ} \mathrm{C}$. This so-called "water-muting temperature" differs from the previously reported value of $4^{\circ} \mathrm{C},{ }^{56}$ perhaps because of a temperature difference between the sample holder and the water in the chip. The THz-OA signals of water below and above the water-muting temperature showed the same waveform inversion as in the megahertz regime [Fig. 3(c)]. ${ }^{56}$

This led us to ask whether the dampening of the water signal would allow isolation of the signal from biomolecular solutes of interest. To address this question, we measured $\mathrm{NaCl}$ aqueous solutions at light concentrations reaching the $\mathrm{NaCl}$ level in the human body. ${ }^{57}$ Time-domain analysis of signals from pure

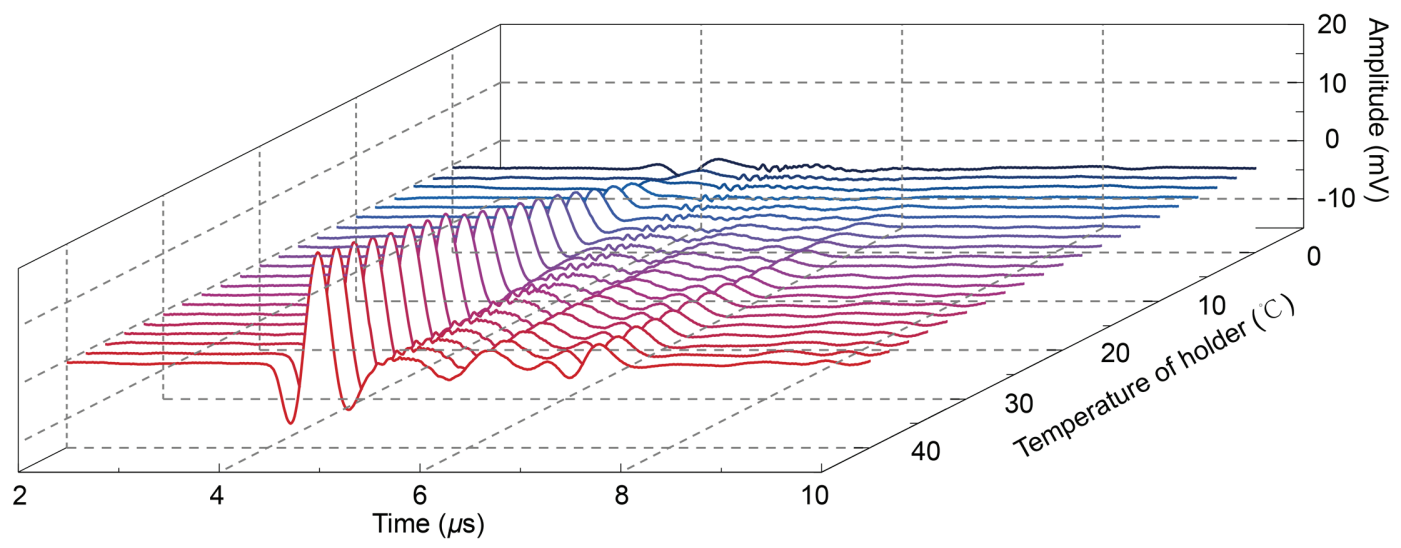

(a)

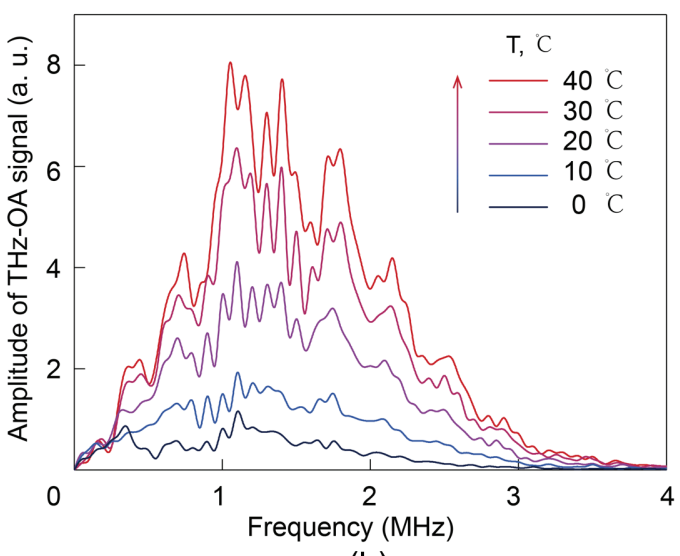

(b)

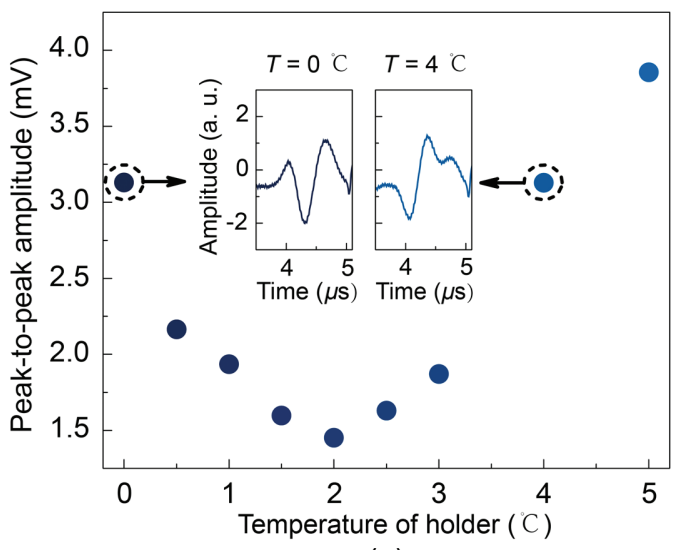

(c)

Fig. 3 Temperature dependence of the THz-OA signal of water. (a) Time-domain THz-OA signal of water at different temperatures. (b) The THz-OA signals from (a) were transformed to the frequency domain. (c) $\mathrm{THz}-\mathrm{OA}$ amplitudes of water over the temperature range from $0^{\circ} \mathrm{C}$ to $5^{\circ} \mathrm{C}$. The insets show the raw $\mathrm{THz}-\mathrm{OA}$ signal at $0^{\circ} \mathrm{C}$ (dark blue) or $4^{\circ} \mathrm{C}$ (light blue). 
water and increasingly concentrated $\mathrm{NaCl}$ solutions showed that the transmitted amplitude measured by the commercially available THz-TDS system cannot be effectively distinguished with concentrations less than $0.5 \mathrm{~mol} / \mathrm{L}$ [Fig. 4(a)], as did the optoacoustic amplitude recorded by our setup at $24^{\circ} \mathrm{C}$ [Fig. 4(b)]. However, the optoacoustic amplitude recorded by our setup at $5^{\circ} \mathrm{C}[\mathrm{Fig}$. 4(c)] increased with $\mathrm{NaCl}$ concentrations, demonstrating that the detection sensitivity can be enhanced to $0.05 \mathrm{~mol} / \mathrm{L}$. The time-domain THz-OA signals also shifted to earlier time points with higher ion concentrations due to changes in the speed of sound in ion solutions. Normalized THz-OA and THz-TDS amplitudes varied with ion concentrations, revealing that the sensitivity of the response was much greater near the water-muting temperature [Fig. 4(d)]. In fact, our time-domain THz-OA method showed an order of magnitude greater sensitivity at $5^{\circ} \mathrm{C}$ than the commercially available $\mathrm{THz}$-TDS system.

With sensitivity-enhanced time-domain terahertz optoacoustics, we also measure aqueous solutions of ions at high concentrations (see Fig. S2 in Supplementary Material) in order to study more characteristic parameters related to both terahertz absorption and ultrasonic propagation. The amplitudes of the $\mathrm{THz}-\mathrm{OA}$ signals from pure water and increasingly concentrated $\mathrm{CaCl}_{2}$ and $\mathrm{NaCl}$ solutions increased with ion concentrations at $5^{\circ} \mathrm{C}$ or $24^{\circ} \mathrm{C}$ when the concentration was lower than the turning point around $4 \mathrm{~mol} / \mathrm{L}$ [Figs. 5(a) and 5(b)]. At the same ion concentration and temperature, the signal amplitude was higher for $\mathrm{CaCl}_{2}$ than $\mathrm{NaCl}$. The difference in signal amplitude between $24^{\circ} \mathrm{C}$ and $5^{\circ} \mathrm{C}$ decreased with increasing ion concentration, which may reflect the compressibility coefficient changing with the increasing number of water molecules located in their hydration sphere. ${ }^{58}$ Since the physical properties of ionic solutions depend on hydration number, ${ }^{59,60}$ both the concentration turning point and compressibility coefficient can be used to quantify the hydration number of aqueous solutions. ${ }^{58,61}$ Our results suggest that the proposed time-domain THz-OA method is indeed promising for examining the hydration number in ionic solutions and its influence on biological activities.

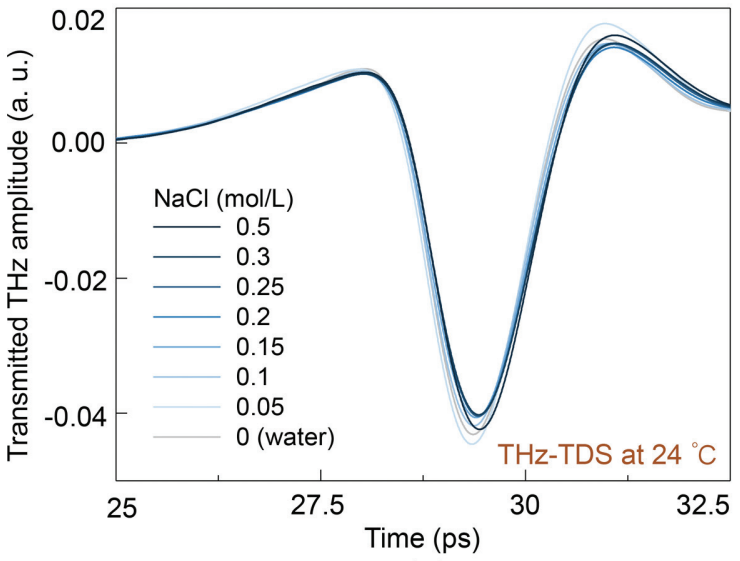

(a)

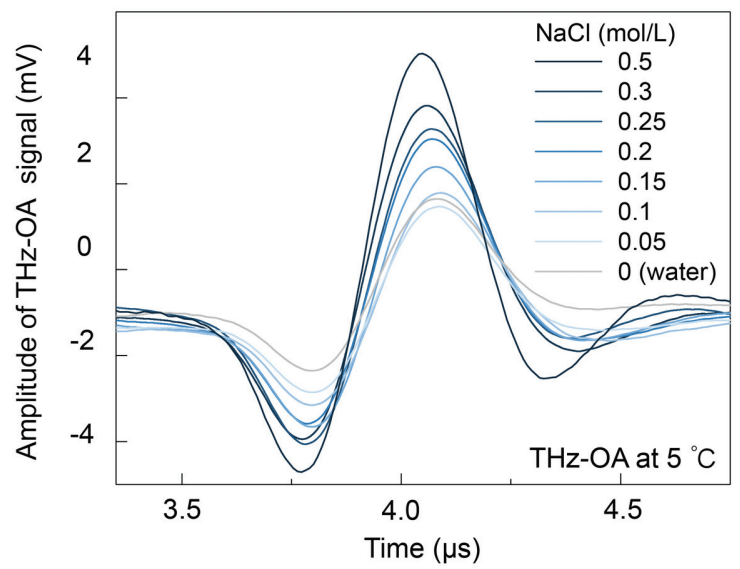

(c)

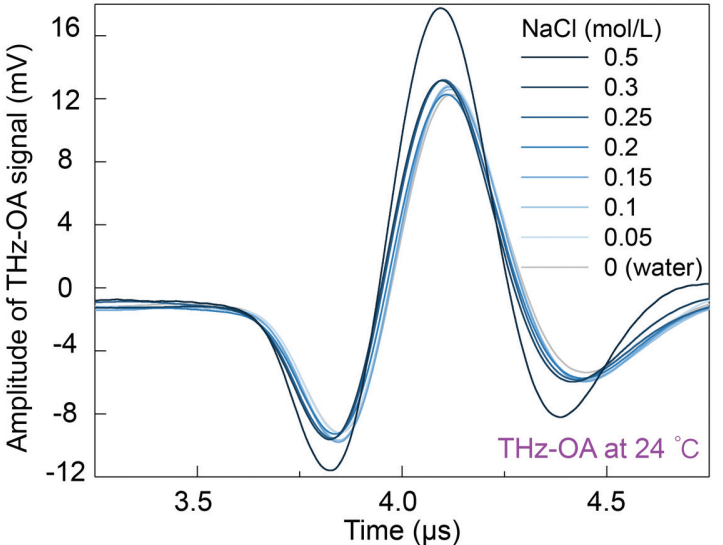

(b)

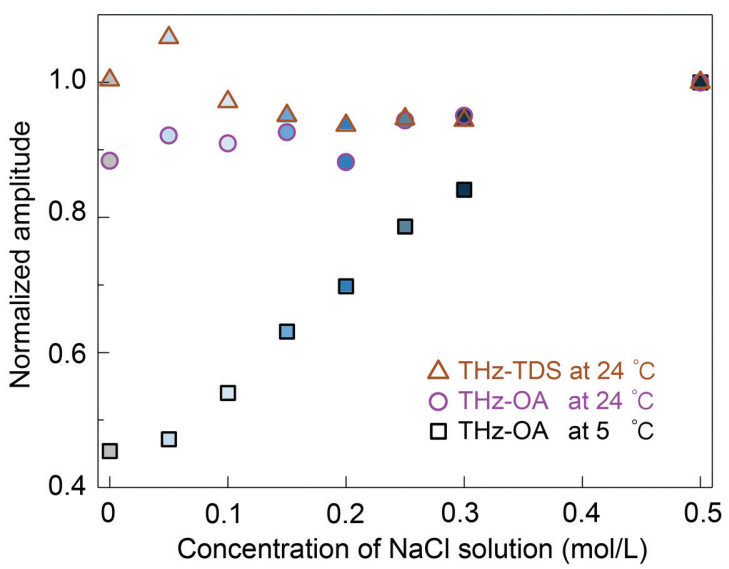

(d)

Fig. 4 Concentration dependence of the time-domain $\mathrm{THz}-\mathrm{OA}$ signal of $\mathrm{NaCl}$ solutions with light concentrations at $24^{\circ} \mathrm{C}$ and $5^{\circ} \mathrm{C}$. (a) THz-TDS profiles of pure water and increasingly concentrated $\mathrm{NaCl}$ solutions. Measurements were taken at $24^{\circ} \mathrm{C}$. (b) $\mathrm{THz}-\mathrm{OA}$ response of pure water and increasingly concentrated $\mathrm{NaCl}$ solutions at $24^{\circ} \mathrm{C}$. (c) The same measurements were performed as in (b) but at $5^{\circ} \mathrm{C}$. (d) Normalized amplitudes obtained for different $\mathrm{NaCl}$ concentrations in aqueous solution using the proposed $\mathrm{THz}-\mathrm{OA}$ effect at $24^{\circ} \mathrm{C}$ (purple) and $5^{\circ} \mathrm{C}$ (black) and a commercially available THz-TDS (brown). 


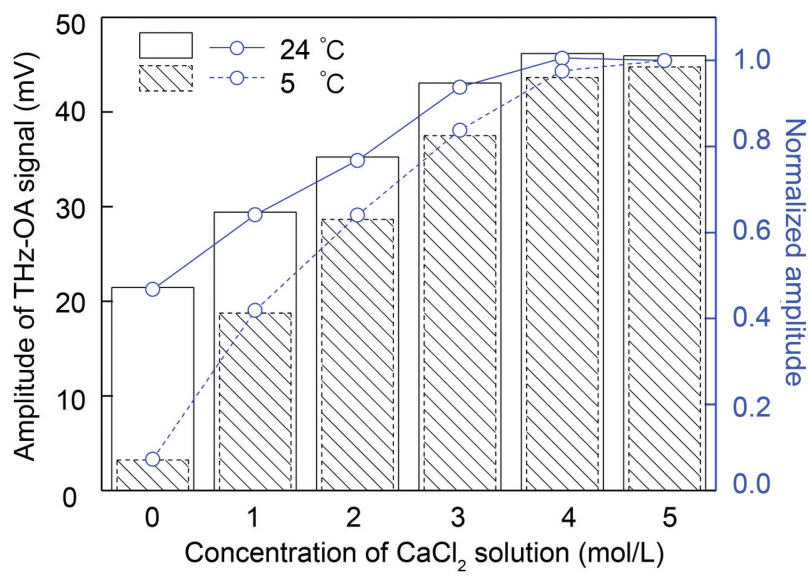

(a)

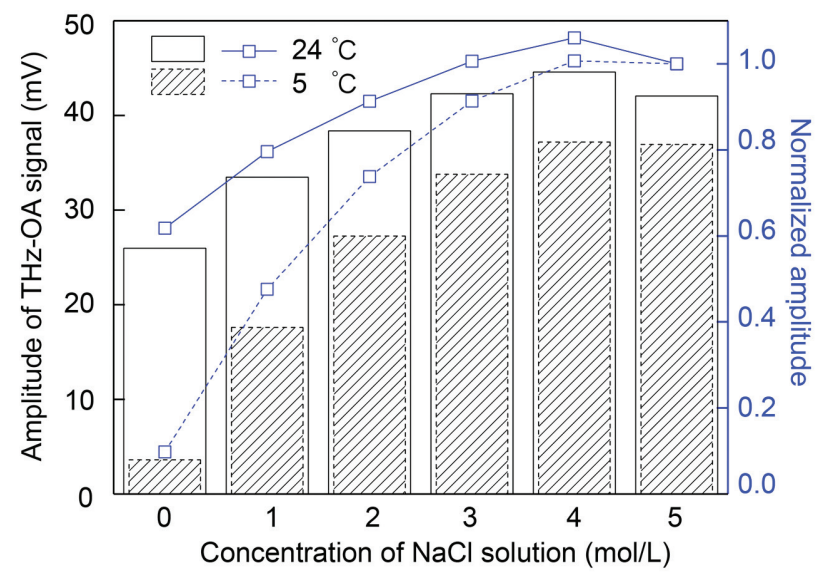

(b)

Fig. 5 Concentration dependence of $\mathrm{THz}-\mathrm{OA}$ amplitude of $\mathrm{CaCl}_{2}$ and $\mathrm{NaCl}$ solutions with high concentrations at $24^{\circ} \mathrm{C}$ and $5^{\circ} \mathrm{C}$. THz-OA amplitude of aqueous solutions of (a) $\mathrm{CaCl}_{2}$ and (b) $\mathrm{NaCl}$ before normalization (gray) and after normalization (blue). Measurements were taken at $24^{\circ} \mathrm{C}$ (solid bar and line) or $5^{\circ} \mathrm{C}$ (dotted bar and line).

\section{Discussion and Conclusion}

Using an ultrafast terahertz source and a conventional piezoelectric transducer, we detected time-domain THz-OA signals from agar-in-water phantoms, ex vivo tissues, as well as water and aqueous solutions in a microfluidic chip over a $10^{4}$-fold range in thickness from microns to centimeters. The THz-OA signal carrying interaction information between terahertz radiation and the sample can be transmitted to a long distance up to several centimeters. Here, the interaction length is longer than that of THz-TDS in reflection or in a thin aqueous sample. This is because all terahertz radiation can be absorbed and converted into the THz-OA signal, i.e., it is not necessary for the terahertz radiation passing through the sample to be detected by the terahertz detector in THz-TDS. The time-domain THz-OA method demonstrated here is a breakthrough that enables detection of THz-OA signals directly induced in water with the thickness $10^{2}$-fold larger than traditional terahertz detection methods, such as THz-TDS. Our observations demonstrate the feasibility of precise and nondestructive THz-OA analysis of thick, biologically relevant samples, eliminating the need for sample pretreatments, such as dehydration or cryosection, which may destroy biological integrity. ${ }^{62,63}$ The experimentally characterized obvious differences in THz-OA signals, both in time and frequency domains, for a range of tissues suggest the potential for spectroscopy and imaging based on time-domain terahertz optoacoustics.

We showed that terahertz optoacoustics can rely on water as a detector of terahertz radiation and that its detection sensitivity can be manipulated by altering temperature. By lowering the temperature of aqueous solutions of ions, we were able to mute the THz-OA contribution from water and enrich it for the contribution from solutes of interest. In fact, our system showed an order of magnitude greater sensitivity to changes in $\mathrm{NaCl}$ concentration than a commercially available THz-TDS system. Our method for the terahertz regime extends previous studies of optoacoustic spectroscopy of aqueous solutions in the near- and mid-infrared regimes, which established the potential of muting the water contribution. ${ }^{56,64}$ This water-manipulated THz-OA method can uniquely achieve sensitivity-enhanced label-free quantification of ion concentrations reaching the concentration level in the human body. More temperature- and concentrationrelated parameters related to both terahertz absorption and ultrasonic propagation can be acquired by THz-OA signals to potentially study the biological and chemical properties, such as the hydration number of ion solutions, through precise mathematical modeling. In addition, we have extended the proposed timedomain terahertz optoacoustics to characterize other biologically important solutes such as glucose and were able to reproduce these experimental results in simulations based on parameters in the literature (see Fig. S3 in Supplementary Material)..$^{51-53}$

The experimental setup in this work relies on a femtosecond laser amplifier and tilted-pulse-front excitation technology, ${ }^{43}$ which generates a more uniform terahertz pulse at safer energies than free-electron lasers. ${ }^{40,41}$ We used terahertz pulses lasting less than one picosecond in order to achieve stable and reproducible optoacoustic response without destructive shockwaves. Such short pulses with reasonable repetition rate and radiant exposure meet the laser limit needed for biological safety, which are unlike the terahertz pulses with excessively high repetition rate or high pulse energy that can influence protein structure or generate bubbles in liquid water. ${ }^{40,41}$

The experimental setup relies on a conventional piezoelectric ultrasonic transducer, which may perform more stably and at lower cost than an optical microring resonator. ${ }^{42}$ The universal ultrasonic transducers have been widely used in biomedical applications to detect both the transparent media and turbid samples, such as biotissues, while the shadowgraph imaging system in Refs. 40 and 41 can only obtain images of transparent samples. In addition, the conventional transducer can be upgraded with multiple channels, allowing simultaneous collection of THz-OA signals from multiple absorbers or even multidimensional THz-OA imaging. Our microfluidic chip was fabricated using PDMS, which allows efficient transmission of THz-OA signals to the detector. ${ }^{42}$ It is possible that derivatives of this polymer or even other materials may increase the intensity of acoustic waves arriving at the detector.

The proposed time-domain THz-OA method can directly reflect interactions between samples and multispectral terahertz radiation, rather than interactions between samples and optoacoustic waves in Refs. 40 and 41. To identify different target 
molecules based on their THz-OA fingerprints, it may be possible to use tunable, narrow-spectrum terahertz radiation sources or custom-designed contrast agents. Tunable terahertz sources would allow multispectral detection as well as functional imaging analogous to multispectral optoacoustic tomography, ${ }^{65-67}$ but with additional information about the composition, structure, and dynamics of inorganic and organic species that the terahertz regime can provide. . $^{-18}$

The ability of this time-domain THz-OA method to span such a large thickness range makes it well suited for optical- and acoustic-resolution microscopy as well as endoscopy. Terahertz optoacoustics offers far more potential than traditional terahertz imaging, in which information is obtained about a super thin layer on the surface of a sample based on reflection or transmission of terahertz radiation relative to a reference signal. In biomedical applications of traditional terahertz imaging, image contrast is strongly limited by the water content of the sample, and spatial resolution is limited by the terahertz diffraction limit. ${ }^{7,31,68}$ Indeed, terahertz optoacoustics offers the same imaging advantages as optoacoustics in other wavelength regimes. Regardless of wavelength, optoacoustics offers much deeper penetration than optical microscopy because acoustic waves are scattered orders of magnitude less than light waves as they pass through the sample. ${ }^{47}$ Another advantage is that optoacoustic signals can contain structural as well as functional information because the initial optoacoustic pressure is related to the functional parameters of samples such as absorption coefficient and thermal expansion coefficient (see Sec. 2).

The time-domain THz-OA method described here may provide a foundation for innovations in spectroscopy and imaging. These include new terahertz-based platforms for biomedicine and chemistry, in particular, such as THz-OA spectroscopy, THz-OA imaging, THz-OA microscopy, THz-OA endoscopy, and multispectral THz-OA functional/molecular imaging.

\section{Acknowledgments}

This work was supported by the National Key Research and Development Program of China (2017YFA0701004), the National Natural Science Foundation of China (61675145, $61722509,81771880,61735012$, and 61420106006), and the Tianjin Municipal Government (19JCQNJC12800). J. L., Y. X. Y., and L. W. J. contributed equally to this work. The authors declare no conflicts of interest.

\section{References}

1. M. Tonouchi, "Cutting-edge terahertz technology," Nat. Photonics 1(2), 97-105 (2007).

2. B. Ferguson and X. Zhang, "Materials for terahertz science and technology," Nat. Mater. 1(1), 26-33 (2002).

3. D. Mittleman, "Frontiers in terahertz sources and plasmonics," Nat. Photonics 7(9), 666-669 (2013).

4. P. Jepsen, D. Cooke, and M. Koch, "Terahertz spectroscopy and imaging-modern techniques and applications," Laser Photonics Rev. 5(1), 124-166 (2011).

5. X. Zhang, A. Shkurinov, and Y. Zhang, "Extreme terahertz science," Nat. Photonics 11(1), 16-18 (2017).

6. E. Pickwell and V. P. Wallace, "Biomedical applications of terahertz technology," J. Phys. D Appl. Phys. 39(17), R301-R310 (2006).

7. X. Yang et al., "Biomedical applications of terahertz spectroscopy and imaging," Trends Biotechnol. 34(10), 810-824 (2016).

8. P. H. Siegel, "Terahertz technology in biology and medicine," IEEE Trans. Microwave Theory 52(10), 2438-2447 (2004).
9. K. A. Niessen et al., "Protein and RNA dynamical fingerprinting," Nat. Commun. 10(1), 1026 (2019).

10. H. J. Shin et al., "Conformational characteristics of beta-glucan in laminarin probed by terahertz spectroscopy," Appl. Phys. Lett. 94(11), 111911 (2009).

11. S. Funkner et al., "Watching the low-frequency motions in aqueous salt solutions: the terahertz vibrational signatures of hydrated ions," J. Am. Chem. Soc. 134(2), 1030-1035 (2012).

12. B. D. Bursulaya and H. J. Kim, "Spectroscopic and dielectric properties of liquid water: a molecular dynamics simulation study," J. Chem. Phys. 109(12), 4911-4919 (1998).

13. J. Xu, K. W. Plaxco, and S. J. Allen, "Probing the collective vibrational dynamics of a protein in liquid water by terahertz absorption spectroscopy," Protein Sci. 15(5), 1175-1181 (2006).

14. B. Born et al., "The terahertz dance of water with the proteins: the effect of protein flexibility on the dynamical hydration shell of ubiquitin," Faraday Discuss. 114, 161-173 (2008).

15. B. Born et al., "Solvation dynamics of model peptides probed by terahertz spectroscopy. Observation of the onset of collective network motions," J. Am. Chem. Soc. 131(10), 3752-3755 (2009).

16. S. Ebbinghaus et al., "An extended dynamical hydration shell around proteins," Proc. Natl. Acad. Sci. U. S. A. 104(52), 20749-20752 (2007).

17. M. C. Bellissent-Funel et al., "Water determines the structure and dynamics of proteins," Chem. Rev. 116(13), 7673-7697 (2016).

18. G. Schwaab, F. Sebastiani, and M. Havenith, "Ion hydration and ion pairing as probed by $\mathrm{THz}$ spectroscopy," Angew. Chem. Int. Ed. 58(10), 3000-3013 (2019).

19. S. J. Kim et al., "Real-time detection of protein-water dynamics upon protein folding by terahertz absorption spectroscopy," Angew. Chem. Int. Ed. 47(34), 6486-6489 (2008).

20. D. A. Turton et al., "Terahertz underdamped vibrational motion governs protein-ligand binding in solution," Nat. Commun. 5(1), 3999 (2014).

21. A. G. Markelz, "Terahertz dielectric sensitivity to biomolecular structure and function," IEEE J. Sel. Top. Quantum 14(1), 180-190 (2008).

22. X. Li et al., "Terahertz absorption of DNA decamer duplex," J. Phys. Chem. A 112(47), 12090-12096 (2008).

23. H. B. Liu et al., "Sensing minute changes in biological cell monolayers with THz differential time-domain spectroscopy," Biosens. Bioelectron. 22(6), 1075-1080 (2007).

24. U. Heugen et al., "Solute-induced retardation of water dynamics probed directly by terahertz spectroscopy," Proc. Natl. Acad. Sci. U. S. A. 103(33), 12301-12306 (2006).

25. M. Heyden et al., "Long-range influence of carbohydrates on the solvation dynamics of water-answers from terahertz absorption measurements and molecular modeling simulations," J. Am. Chem. Soc. 130(17), 5773-5779 (2008).

26. N. Penkov et al., "Terahertz spectroscopy applied for investigation of water structure," J. Phys. Chem. B 119(39), 12664-12670 (2015).

27. H. Chen et al., "Performance of THz fiber-scanning near-field microscopy to diagnose breast tumors," Opt. Express 19(20), 19523-19531 (2011).

28. H. Cheon et al., "Detection and manipulation of methylation in blood cancer DNA using terahertz radiation," Sci. Rep. 9(1), 6413 (2019).

29. W. G. Yeo et al., "Evaluation of cancer tissue morphology via $\mathrm{THz}$ spectroscopic imaging: human lung and small intestine malignancies," Infrared Phys. Technol. 97, 411-416 (2019).

30. M. El-Shenawee et al., "Cancer detection in excised breast tumors using terahertz imaging and spectroscopy," Biomed. Spectrosc. Imaging 8(1-2), 1-9 (2019).

31. Z. Geng et al., "A route to terahertz metamaterial biosensor integrated with microfluidics for liver cancer biomarker testing in early stage," Sci. Rep. 7(1), 16378 (2017).

32. E. Pickwell et al., "In vivo study of human skin using pulsed terahertz radiation," Phys. Med. Biol. 49(9), 1595-1607 (2004). 
33. P. Tewari et al., "In vivo terahertz imaging of rat skin burns," J. Biomed. Opt. 17(4), 040503 (2012).

34. P. C. Ashworth et al., "Terahertz pulsed spectroscopy of freshly excised human breast cancer," Opt. Express 17(15), 12444 (2009).

35. L. Thrane et al., "Thz reflection spectroscopy of liquid water," Chem. Phy. Lett. 240(4), 330-333 (1995).

36. T. Arikawa, M. Nagai, and K. Tanaka, "Characterizing hydration state in solution using terahertz time-domain attenuated total reflection spectroscopy," Chem. Phy. Lett. 457(1-3), 12-17 (2008).

37. M. A. Brun et al., "Terahertz imaging applied to cancer diagnosis," Phys. Med. Biol. 55(16), 4615-4623 (2010).

38. H. A. Hafez et al., "Intense terahertz radiation and their applications," J. Opt. 18(9), 093004 (2016).

39. X. Cai et al., "Sensitive room-temperature terahertz detection via the photothermoelectric effect in graphene," Nat. Nanotechnol. 9(10), 814-819 (2014).

40. M. Tsubouchi et al., "Plane photoacoustic wave generation in liquid water using irradiation of terahertz pulses," Sci. Rep. 10(1), 18537 (2020).

41. S. Yamazaki et al., "Propagation of $\mathrm{THz}$ irradiation energy through aqueous layers: demolition of actin filaments in living cells," Sci. Rep. 10(1), 9008 (2020).

42. S. L. Chen et al., "Efficient real-time detection of terahertz pulse radiation based on photoacoustic conversion by carbon nanotube nanocomposite," Nat. Photonics 8(7), 537-542 (2014).

43. J. Hebling et al., "Generation of high-power terahertz pulses by tilted-pulse-front excitation and their application possibilities," J. Opt. Soc. Am. B 25(7), B6-B19 (2008).

44. Y. Zhou et al., "Tutorial on photoacoustic tomography," J. Biomed. Opt. 21(6), 061007 (2016).

45. ANSI, "American national standard for safe use of lasers," Laser Institute of America ANSI Z136.1 (2007).

46. J. Yao and L. V. Wang, "Photoacoustic microscopy," Laser Photonics Rev. 7(5), 758-778 (2013).

47. V. Ntziachristos, "Going deeper than microscopy: the optical imaging frontier in biology," Nat. Methods 7(8), 603-614 (2010).

48. L. V. Wang and H. I. Wu, Biomedical Optics Principles and Imaging, Ch. 12, Wiley Press, New Jersey (2009).

49. L. V. Wang, "Tutorial on photoacoustic microscopy and computed tomography," IEEE J. Sel. Top. Quantum 14(1), 171-179 (2008).

50. B. E. Treeby and B. T. Cox, "k-Wave: MATLAB toolbox for the simulation and reconstruction of photoacoustic wave fields," J. Biomed. Opt. 15(2), 021314 (2010).

51. R. Darros-Barbosa, M. O. Balaban, and A. A. Teixeira, "Temperature and concentration dependence of density of model liquid foods," Int. J. Food Prop. 6(2), 195-214 (2003).

52. N. Contreras et al., "Analysis of the sugar content of fruit juices and drinks using ultrasonic velocity measurements," Int. J. Food Sci. Technol. 27(5), 515-529 (1992).

53. R. Darros-Barbosa, "High pressure and temperature dependence of thermodynamic properties of model food solutions obtained from in situ ultrasonic measurements," $\mathrm{PhD}$ Thesis, University of Florida, Gainesville, Florida (2003).

54. C Huang et al., "Photoacoustic computed tomography correcting for heterogeneity and attenuation," J. Biomed. Opt. 17(6), 061211 (2012).

55. T. Lu et al., "Full-frequency correction of spatial impulse response in back-projection scheme using space-variant filtering for optoacoustic mesoscopy," Photoacoustics 19, 100193 (2020).

56. J. Prakash et al., "Short-wavelength optoacoustic spectroscopy based on water muting," Proc. Natl. Acad. Sci. U. S. A. 117(8), 4007-4014 (2020).

57. D. S. Wishart et al., "HMDB 4.0: the human metabolome database for 2018," Nucleic Acids Res. 46 (D1), D608-D617 (2018).

58. A. Burakowski and J. Gliński, "Hydration numbers of nonelectrolytes from acoustic methods," Chem. Rev. 112(4), 2059-2081 (2012).
59. K. J. Tielrooij et al., "Cooperativity in ion hydration," Science 328(5981), 1006-1009 (2010).

60. Y. Marcus, "Effect of ions on the structure of water: structure making and breaking," Chem. Rev. 109(3), 1346-1370 (2009).

61. M. Z. Jora, M. V. C. Cardoso, and E. Sabadini, "Dynamical aspects of water-poly(ethylene glycol) solutions studied by ${ }^{1} \mathrm{H}$ NMR," J. Mol. Liq. 222, 94-100 (2016).

62. A. S. Kolesnikov et al., "In vitro terahertz monitoring of muscle tissue dehydration under the action of hyperosmotic agents," Quantum Electron. 44(7), 633-640 (2014).

63. O.A. Smolyanskaya et al., "Terahertz biophotonics as a tool for studies of dielectric and spectral properties of biological tissues and liquids," Prog. Quant. Electron. 62, 1-77 (2018).

64. J. Shi et al., "High-resolution, high-contrast mid-infrared imaging of fresh biological samples with ultraviolet-localized photoacoustic microscopy," Nat. Photonics 13(9), 609-615 (2019).

65. S. Tzoumas et al., "Unmixing molecular agents from absorbing tissue in multispectral optoacoustic tomography," IEEE Trans. Med. Imaging 33(1), 48-60 (2014).

66. B. Cox et al., "Quantitative spectroscopic photoacoustic imaging: a review," J. Biomed. Opt. 17(6), 061202 (2012).

67. V. Ntziachristos and D. Razansky, "Molecular imaging by means of multispectral optoacoustic tomography (MSOT)," Chem. Rev. 110(5), 2783-2794 (2010).

68. J. Kappa et al., "Electrically reconfigurable micromirror array for direct spatial light modulation of terahertz waves over a bandwidth wider than 1 THz," Sci. Rep. 9(1), 2597 (2019).

Jiao $\mathbf{L i}$ is an associate professor at Tianjin University. She received her $\mathrm{PhD}$ in biomedical engineering from Tianjin University in 2013, and, after that, she joined the School of Precision Instrument and Optoelectronics Engineering of Tianjin University. From 2015 to 2016, she was a visiting scholar at the Helmholtz Zentrum in Munich, Germany. She is the author of more than 30 journal papers. Her current research interests include multispectrum multiscale optoacoustic imaging and advanced optical molecular imaging with various functional nanomaterials and nanotechnologies for studying biological processes and diseases.

Yixin Yao is a graduate student at Tianjin University. She received her BEng degree in opto-electronics technology from Tianjin University, China, in 2019. She will enter the PhD program in optical engineering at Tianjin University in fall 2021. Her research interests are terahertz spectroscopy and imaging.

Liwen Jiang is a PhD student at Tianjin University majoring in opto-electronics technology. She received her MS degree in IC engineering from the Institute of Microelectronics, of the Chinese Academy of Sciences, in 2018. Her research interests are terahertz spectroscopy and microscopy.

Zhen Tian is a full professor at Tianjin University, China. He received his BEng degree in electronic science and technology, MEng degree in physical electronics, and $\mathrm{PhD}$ in optical engineering from Tianjin University, China, in 2004, 2007, and 2010, respectively. Up to now, he has published over 100 peer-reviewed journal papers with total citations of $\sim 2700$ ( $\mathrm{H}$ index is 28). His current research interests focus on ultrafast terahertz spectroscopy and terahertz subwavelength devices.

Weili Zhang is a full professor at Oklahoma State University. He received his PhD in optical engineering from Tianjin University, China, in 1993. $\mathrm{He}$ was a postdoctoral research associate at the Hong Kong University of Science and Technology from 1993 to 1995. His research interests include terahertz optoelectronics, nano- and microstructured materials optics, and ultrafast phenomena. He is a fellow of OSA.

Biographies of the other authors are not available. 